\title{
Technical Note: Approximate solution of transient drawdown for constant-flux pumping at a partially penetrating well in a radial two-zone confined aquifer
}

\author{
C.-S. Huang ${ }^{1}$, S.-Y. Yang ${ }^{2}$, and H.-D. Yeh ${ }^{1}$ \\ ${ }^{1}$ Institute of Environmental Engineering, National Chiao Tung University, Hsinchu, Taiwan \\ ${ }^{2}$ Department of Civil Engineering, Vanung University, Chungli, Taiwan
}

Correspondence to: H.-D. Yeh (hdyeh@mail.nctu.edu.tw)

Received: 5 February 2015 - Published in Hydrol. Earth Syst. Sci. Discuss.: 4 March 2015

Accepted: 16 May 2015 - Published: 5 June 2015

\begin{abstract}
An aquifer consisting of a skin zone and a formation zone is considered as a two-zone aquifer. Existing solutions for the problem of constant-flux pumping in a two-zone confined aquifer involve laborious calculation. This study develops a new approximate solution for the problem based on a mathematical model describing steady-state radial and vertical flows in a two-zone aquifer. Hydraulic parameters in these two zones can be different but are assumed homogeneous in each zone. A partially penetrating well may be treated as the Neumann condition with a known flux along the screened part and zero flux along the unscreened part. The aquifer domain is finite with an outer circle boundary treated as the Dirichlet condition. The steady-state drawdown solution of the model is derived by the finite Fourier cosine transform. Then, an approximate transient solution is developed by replacing the radius of the aquifer domain in the steady-state solution with an analytical expression for a dimensionless time-dependent radius of influence. The approximate solution is capable of predicting good temporal drawdown distributions over the whole pumping period except at the early stage. A quantitative criterion for the validity of neglecting the vertical flow due to a partially penetrating well is also provided. Conventional models considering radial flow without the vertical component for the constant-flux pumping have good accuracy if satisfying the criterion.
\end{abstract}

\section{Introduction}

The constant-flux pumping (CFP) test is a widely used well test for characterizing the aquifer properties such as transmissivity and storage coefficient. The test is performed with a constant pumping rate at a fully or partially penetrating well in either a confined or unconfined aquifer. Existing analytical solutions for the CFP in a homogenous confined aquifer are briefly reviewed herein. Theis (1935) was the first article in the groundwater literature to present an analytical solution for aquifer drawdown due to pumping in a fully penetrating well with an infinitesimal radius. Carslaw and Jaeger (1959) presented analytical solutions for the three kinds of heat conduction problems which can be analogous to the CFP problems including the aquifers of the infinite domain with a finite-radius well, finite domain with a finiteradius well, and finite domain with an infinitesimal-radius well. Hantush (1962) developed an analytical solution of drawdown induced by a partially penetrating well for the CFP. Papadopulos and Cooper (1967) obtained an analytical solution of drawdown considering the effects of well radius and wellbore storage. They provided a quantitative criterion of time for neglecting the effects. The criterion will be stated in the next section. Chen (1984) derived an analytical solution for drawdown in a circular aquifer with the Dirichlet boundary condition of zero drawdown and provided a quantitative criterion describing the beginning time of the boundary effect on the drawdown. Yang et al. (2006) developed an analytical solution describing aquifer drawdown due to a partially penetrating well with a finite radius. The effect of partial penetration on temporal drawdown distributions was 
discussed. Wang and Yeh (2008) provided a quantitative criterion for the beginning time of the boundary effect on drawdown induced by the CFP and constant-head pumping. Yeh and Chang (2013) provided a comprehensive review on analytical solutions for the CFP in unconfined and multilayered aquifer systems.

Drilling an aquifer to install a well may decrease or increase the permeability of the formation around the wellbore. The perturbed formation, called skin zone, extends from a few millimeters to several meters. A positive skin zone means that its permeability is lower than the original formation. On the other hand, a negative skin zone is of a higher permeability than the original formation. Existing solutions accounting for the CFP in a two-zone confined aquifer consisting of the skin zone and formation zone are reviewed. Novakowski (1989) developed a semi-analytical solution of drawdown with the wellbore storage effect and investigated the effect of an infinitesimally thin skin on temporal drawdown curves. Hemker (1999) proposed an analyticalnumerical solution describing pumping drawdown in a multilayered aquifer system where the radial flow was analytically treated and the vertical one was handled by a finite difference method. The flux along the well screen was non-uniform through an infinitesimal thin skin, and the flow was subject to the wellbore storage effect. Kabala and El-Sayegh (2002) presented a semi-analytical solution for the transient flowmeter test in a multilayered aquifer system where the radial flow was considered in each layer assuming no vertical flow component and uniform flux along the well screen. Predictions from the solution were compared with those from a numerical solution which relaxes those two assumptions. Yeh et al. (2003) obtained an analytical solution for pumping drawdown induced by a finite-radius well in a two-zone confined aquifer and discussed the error caused by neglecting the well radius. Chen and Chang (2006) developed a semi-analytical solution for the CFP on the basis of the Gram-Schmidt method to deal with the non-uniform skin effect represented by an arbitrary piecewise function of elevation. They indicated that flow near a pumping well is three dimensional due to the effect and away from the well is radial. Perina and Lee (2006) proposed a general well function for transient flow toward a partially penetrating well considering the wellbore storage effect and non-uniform flux between the screen and skin zone in a confined, unconfined, or leaky aquifer. Chiu et al. (2007) developed a semi-analytical solution for the CFP at a partially penetrating well in a two-zone confined aquifer. They indicated that the influence of the partial penetration on drawdown is more significant for a negative skin zone than a positive one. C. T. Wang et al. (2012) provided an analytical solution of drawdown for the CFP in a twozone confined aquifer of finite extent with an outer boundary under the Dirichlet condition of zero drawdown. They also derived a large-time drawdown solution which reduces to the Thiem solution in the absence of the skin zone. X. Wang et al. (2012) presented a finite layer method (FLM) based on
Galerkin's technique for simulating radial and vertical flows toward a partially penetrating well in a multilayered aquifer system. The FLM was verified by an analytical solution and finite difference solution.

It is informative to classify the above solutions into two groups, i.e., homogeneous aquifer and two-zone aquifer systems in Table 1. The solutions in each group are categorized according to the well penetration, well radius, and wellbore storage.

At present, a time-domain analytical solution of drawdown for flow induced by the CFP at a finite-radius partially penetrating well in a two-zone confined aquifer has not been developed. The Laplace-domain result of the above-mentioned problem was presented by Chiu et al. (2007) resorting to a numerical inversion scheme called the Crump method. The application of their solution may therefore be inconvenient for those who are not familiar with numerical approaches. The purpose of this note is to develop a new approximate transient solution for the problem in a way similar to the work of Yang et al. (2014). A mathematical model for steadystate flow due to a partially penetrating well in a finite-extent two-zone confined aquifer is built. The flow equations describing spatial drawdowns in the skin and formation zones are employed. The outer boundary of the aquifer is specified as the Dirichlet condition of zero drawdown. The well is treated as the Neumann condition with a constant flux for the screened part and zero flux for the unscreened part. The steady-state solution of the model for drawdown is derived by the method of finite Fourier cosine transform. The approximate transient solution of drawdown is then obtained on the basis of the steady-state solution and a time-dependent radius of influence. The transient solution is in terms of simple series with advantages of fast convergence, simplicity, and good accuracy from a practical viewpoint. It can be used as a convenient tool to estimate temporal and spatial drawdown distributions for the constant-flux pumping and to explore physical insights into the flow behavior affected by hydrogeological properties and aquifer configuration. The accuracy of the solution is investigated in comparison with the Chiu et al. (2007) solution. In addition, the condition of neglecting the effect of the vertical flow on temporal drawdown distributions is investigated.

\section{Methodology}

\subsection{Mathematical model}

This section introduces a new mathematical model for steady-state flow due to the CFP at a finite-radius partially penetrating well in a radial two-zone confined aquifer. The symbols representing variables and parameters for the model are listed in Table A1. The hydraulic parameters in the two zones are different but in each zone are assumed homogeneous. The outer boundary is considered to be un- 
Table 1. Categorization of the solutions for the constant-flux pumping in confined aquifers.

\begin{tabular}{|c|c|c|c|c|}
\hline References & $\begin{array}{l}\text { Well } \\
\text { penetration }\end{array}$ & $\begin{array}{l}\text { Well } \\
\text { radius }\end{array}$ & $\begin{array}{l}\text { Wellbore } \\
\text { storage }\end{array}$ & Remark \\
\hline \multicolumn{5}{|c|}{ Homogeneous aquifer } \\
\hline Theis $(1935)^{\mathrm{a}}$ & Fully & Infinitesimal & None & Infinite aquifer \\
\hline Carslaw and Jaeger $(1959, \text { p. } 328)^{\mathrm{a}}$ & Fully & Finite & None & Infinite aquifer \\
\hline Carslaw and Jaeger $\left(1959\right.$, p. 332) ${ }^{\mathrm{a}}$ & Fully & Finite & None & Finite aquifer with Dirichlet boundary \\
\hline Carslaw and Jaeger $(1959, \text { p. } 335)^{\mathrm{a}}$ & Fully & Infinitesimal & None & Finite aquifer with Dirichlet boundary \\
\hline Hantush $(1962)^{\mathrm{a}}$ & Partially & Infinitesimal & None & Infinite aquifer \\
\hline Papadopulos and Cooper $(1967)^{\mathrm{a}}$ & Fully & Finite & Considered & Infinite aquifer \\
\hline Chen $(1984)^{\mathrm{a}}$ & Fully & Infinitesimal & None & Finite aquifer with Dirichlet boundary \\
\hline Yang et al. $(2006)^{\mathrm{a}}$ & Partially & Finite & None & Infinite aquifer \\
\hline \multicolumn{5}{|c|}{ Two-zone aquifer } \\
\hline Novakowski (1989) ${ }^{b}$ & Fully & Finite & Considered & Infinite aquifer \\
\hline Hemker (1999) ${ }^{\mathrm{c}, *}$ & Partially & Finite & Considered & Multilayered aquifer with radial and vertical flows \\
\hline Kabala and El-Sayegh $(2002)^{\mathrm{b}}$ & Fully & Finite & Considered & Multilayered aquifer with radial flow only \\
\hline Yeh et al. $(2003)^{a}$ & Fully & Finite & None & Infinite aquifer \\
\hline Chen and Chang $(2006)^{b, *}$ & Fully & Finite & Considered & Non-uniform skin effect \\
\hline Perina and Lee $(2006)^{b}$ & Partially & Finite & Considered & General well functions for three-kinds of aquifers \\
\hline Chiu et al. (2007) ${ }^{\mathrm{b}}$ & Partially & Finite & None & Infinite aquifer \\
\hline C. T. Wang et al. (2012) ${ }^{\mathrm{a}}$ & Fully & Finite & None & Finite aquifer with Dirichlet boundary \\
\hline X. Wang et al. $(2012)^{\mathrm{a}}$ & Partially & Infinitesimal & None & Multilayered aquifer with radial and vertical flows \\
\hline
\end{tabular}

${ }^{a},{ }^{b}$ and ${ }^{c}$ represent analytical, semi-analytical and analytical-numerical solutions, respectively. ${ }^{*}$ represents an infinitesimal thin skin zone.

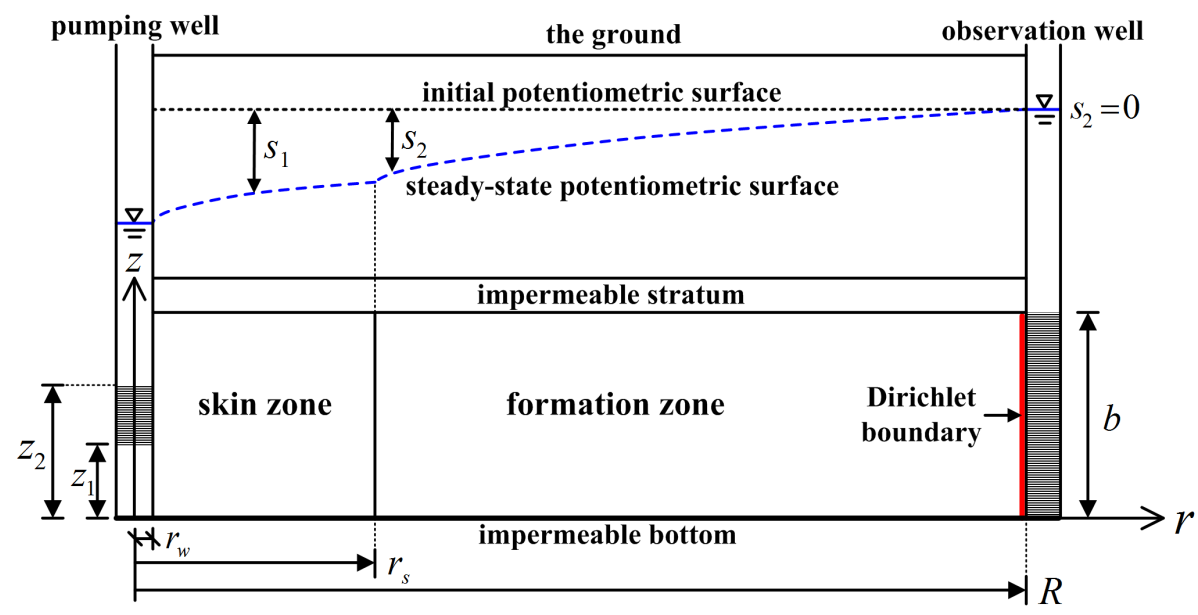

Figure 1. A schematic diagram of the constant-flux pumping at a partially penetrating well in a cylinder two-zone confined aquifer with the Dirichlet boundary. (The symbols of the variables are defined in Table 2.)

der the Dirichlet condition of $\bar{s}_{2}=0$ at $\bar{r}=\bar{R}$. The top and bottom confining beds are under the no-flow conditions of $\partial \bar{s}_{i} / \partial \bar{z}=0$ where $i \in(1,2)$. The effect of wellbore storage on aquifer drawdown is assumed ignorable. Note that this effect diminishes when $t>2.5 \times 10^{2} r_{\mathrm{c}}^{2} / T_{2}$, as mentioned in Papadopulos and Cooper (1967). In addition, Yeh and Chang (2013) also mentioned that this effect can be neglected for a well with $r_{\mathrm{c}} \leq 0.25 \mathrm{~m}$. A schematic diagram for the CFP problem is illustrated in Fig. 1.
The governing equations describing steady-state dimensionless drawdown distributions in the skin and formation zones are expressed, respectively, as

$\frac{\partial^{2} \bar{s}_{1}}{\partial \bar{r}^{2}}+\frac{1}{\bar{r}} \frac{\partial \bar{s}_{1}}{\partial \bar{r}}+\alpha_{1} \frac{\partial^{2} \bar{s}_{1}}{\partial \bar{z}^{2}}=0$ for $1 \leq \bar{r} \leq \bar{r}_{\mathrm{s}}$,

and 
$\frac{\partial^{2} \bar{s}_{2}}{\partial \bar{r}^{2}}+\frac{1}{\bar{r}} \frac{\partial \bar{s}_{2}}{\partial \bar{r}}+\alpha_{2} \frac{\partial^{2} \bar{s}_{2}}{\partial \bar{z}^{2}}=0$ for $\bar{r}_{\mathrm{s}} \leq \bar{r} \leq \bar{R}$,

where $\alpha_{1}$ and $\alpha_{2}$ reflect the effect of aquifer anisotropy on dimensionless aquifer drawdown. The inner boundary designated at the rim of the wellbore is under the Neumann condition as

$$
\begin{gathered}
\frac{\partial \bar{s}_{1}}{\partial \bar{r}}=-\frac{\gamma}{\varphi}\left(U\left(\bar{z}-\bar{z}_{1}\right)-U\left(\bar{z}-\bar{z}_{2}\right)\right) \\
\text { at } \bar{r}=1 \text { and } 0 \leq \bar{z} \leq 1,
\end{gathered}
$$

where $U(\cdot)$ is the unit step function. Equation (3) indicates that the flux is uniformly distributed over the screen. Two continuity conditions required at $\bar{r}=\bar{r}_{\mathrm{s}}$ are

$\bar{s}_{1}=\bar{s}_{2}$ at $\bar{r}=\bar{r}_{\mathrm{s}}$,

and

$$
\frac{\partial \bar{s}_{1}}{\partial \bar{r}}=\gamma \frac{\partial \bar{s}_{2}}{\partial \bar{r}} \text { at } \bar{r}=\bar{r}_{\mathrm{s}} .
$$

\subsection{Steady-state solution}

A new solution derived by the application of the finite Fourier cosine transform to the model can be written as

$$
\begin{aligned}
s_{1}(\bar{r}, \bar{z})= & \ln \left(\bar{R} / \bar{r}_{\mathrm{s}}\right)+\gamma \ln \left(\bar{r}_{\mathrm{s}} / \bar{r}\right)+\frac{2 \gamma}{\bar{z}_{2}-\bar{z}_{1}} \\
& \sum_{n=1}^{\infty} F_{1}(\bar{r}, n) \cos (n \pi \bar{z}) \text { for } 1 \leq \bar{r} \leq \bar{r}_{\mathrm{s}},
\end{aligned}
$$

and

$$
\begin{aligned}
s_{2}(\bar{r}, \bar{z})= & \ln (\bar{R} / \bar{r})+\frac{2 \gamma}{\bar{r}_{\mathrm{s}}\left(\bar{z}_{2}-\bar{z}_{1}\right)} \\
& \sum_{n=1}^{\infty} F_{2}(\bar{r}, n) \cos (n \pi \bar{z}) \text { for } \bar{r}_{\mathrm{s}} \leq \bar{r} \leq \bar{R},
\end{aligned}
$$

with

$$
\begin{aligned}
& F_{1}(\bar{r}, n)=\omega\left(\zeta I_{0}\left(\lambda_{1} \bar{r}\right)+\xi K_{0}\left(\lambda_{1} \bar{r}\right)\right) /\left(\lambda_{1} \psi\right), \\
& F_{2}(\bar{r}, n)=\omega\left(K_{0}\left(\lambda_{2} \bar{R}\right) I_{0}\left(\lambda_{2} \bar{r}\right)-I_{0}\left(\lambda_{2} \bar{R}\right) K_{0}\left(\lambda_{2} \bar{r}\right)\right) /\left(\lambda_{1} \psi\right), \\
& \psi=\lambda_{1} G(0,-1) H(1,-1)-\gamma \lambda_{2} G(1,1) H(0,1), \\
& \zeta=\lambda_{1} K_{1}\left(\lambda_{1} \bar{r}_{\mathrm{s}}\right) G(0,-1)+\gamma \lambda_{2} K_{0}\left(\lambda_{1} \bar{r}_{\mathrm{s}}\right) G(1,1), \\
& \xi=\lambda_{1} I_{1}\left(\lambda_{1} \bar{r}_{\mathrm{s}}\right) G(0,-1)-\gamma \lambda_{2} I_{0}\left(\lambda_{1} \bar{r}_{\mathrm{s}}\right) G(1,1), \\
& G(\mu, c)=I_{\mu}\left(\lambda_{2} \bar{r}_{\mathrm{s}}\right) K_{0}\left(\lambda_{2} \bar{R}\right)+c K_{\mu}\left(\lambda_{2} \bar{r}_{\mathrm{s}}\right) I_{0}\left(\lambda_{2} \bar{R}\right), \\
& H(\mu, c)=K_{1}\left(\lambda_{1}\right) I_{\mu}\left(\lambda_{1} \bar{r}_{\mathrm{s}}\right)+c I_{1}\left(\lambda_{1}\right) K_{\mu}\left(\lambda_{1} \bar{r}_{\mathrm{s}}\right),
\end{aligned}
$$

and

$\omega=\left(\sin \left(\bar{z}_{2} \pi n\right)-\sin \left(\bar{z}_{1} \pi n\right)\right) /(\pi n)$,

where $\lambda_{i}=\pi n \sqrt{\alpha_{i}}$, and $I_{\mu}(\cdot)$ and $K_{\mu}(\cdot)$ are the modified Bessel functions of the first and second kinds with order $\mu$, respectively. The detailed derivation of the solution is given in Appendix A.

\subsection{Approximate transient solution}

The inverse Laplace transform to Chiu et al. (2007) semianalytical solution of drawdown leads to a time-domain result for the CFP in a two-zone aquifer system; however, the resultant solution involves laborious calculations. We therefore develop an approximate transient solution of drawdown for the CFP problem. The idea originated from the concept of a time-dependent diffusion layer for the solution of the diffusion equation in the field of electrochemistry (Fang et al., 2009). The approximate transient solution is obtained by replacing the $\bar{R}$ in the steady-state solution (i.e., Eqs. $6-$ $15)$ with a dimensionless time-dependent radius of influence $\bar{R}(\bar{t})$. The result is in terms of dimensionless time denoted as

$$
\begin{aligned}
s_{1}(\bar{r}, \bar{z}, \bar{t})= & \ln \left(\bar{R}(\bar{t}) / \bar{r}_{\mathrm{s}}\right)+\gamma \ln \left(\bar{r}_{\mathrm{s}} / \bar{r}\right)+\frac{2 \gamma}{\bar{z}_{2}-\bar{z}_{1}} \\
& \sum_{n=1}^{\infty} F_{1}(\bar{r}, \bar{t}) \cos (n \pi \bar{z}) \text { for } 1 \leq \bar{r} \leq \bar{r}_{\mathrm{s}}, \\
s_{2}(\bar{r}, \bar{z}, \bar{t})= & \ln (\bar{R}(\bar{t}) / \bar{r})+\frac{2 \gamma}{\bar{r}_{\mathrm{s}}\left(\bar{z}_{2}-\bar{z}_{1}\right)} \\
& \sum_{n=1}^{\infty} F_{2}(\bar{r}, n, \bar{t}) \cos (n \pi \bar{z}) \text { for } \bar{r}_{\mathrm{s}} \leq \bar{r} \leq \bar{R},
\end{aligned}
$$

and

$\bar{R}(\bar{t})=1+\sqrt{\pi \bar{t} / 1.4}$,

where $F_{1}(\bar{r}, n, \bar{t})$ and $F_{2}(\bar{r}, n, \bar{t})$ obtained from Eqs. (8) and (9), respectively, with coefficients $\psi, \zeta, \xi$, and $G(\mu, c)$ defined in Eqs. (10)-(13), respectively, are functions of dimensionless time due to substitution of Eq. (18). The timedependent radius of influence $\bar{R}(\bar{t})$ was first assumed as $\bar{R}(\bar{t})=1+\sqrt{\pi \bar{t} / c}$ where $c$ is a constant. By trial and error, we found that the drawdowns predicted by the approximate solution and Chiu et al. (2007) Laplace-domain solution with the Crump method agree well when $c$ approaches 1.4. A detailed discussion follows in Sect. 3.1. Notice that Eq. (18) is similar to an equation given in Yang et al. (2014, Eq. 25) but has a different coefficient value.

\subsection{Special case 1: solution for CFP at fully penetrating well in a two-zone aquifer}

When $\bar{z}_{1}=0$ and $\bar{z}_{2}=1$ (i.e., $z_{1}=0$ and $z_{2}=b$ ) for the case of well full penetration, one can obtain $\omega=0$ according to Eq. (15). The simple series in Eqs. (16) and (17) then vanishes, and the solution for temporal drawdown distributions subject to the skin effect reduces to

$s_{1}(\bar{r}, \bar{t})=\ln \left(\bar{R}(\bar{t}) / \bar{r}_{\mathrm{s}}\right)+\gamma \ln \left(\bar{r}_{\mathrm{s}} / \bar{r}\right)$ for $1 \leq \bar{r} \leq \bar{r}_{\mathrm{s}}$,

and

$s_{2}(\bar{r}, \bar{t})=\ln (\bar{R}(\bar{t}) / \bar{r})$ for $\bar{r}_{\mathrm{s}} \leq \bar{r} \leq \bar{R}(\bar{t})$. 
(a)

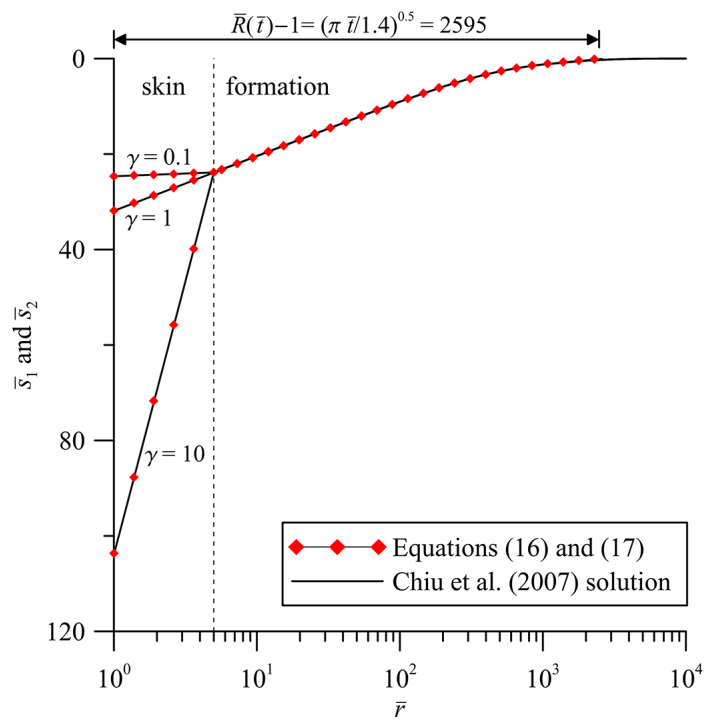

(b)

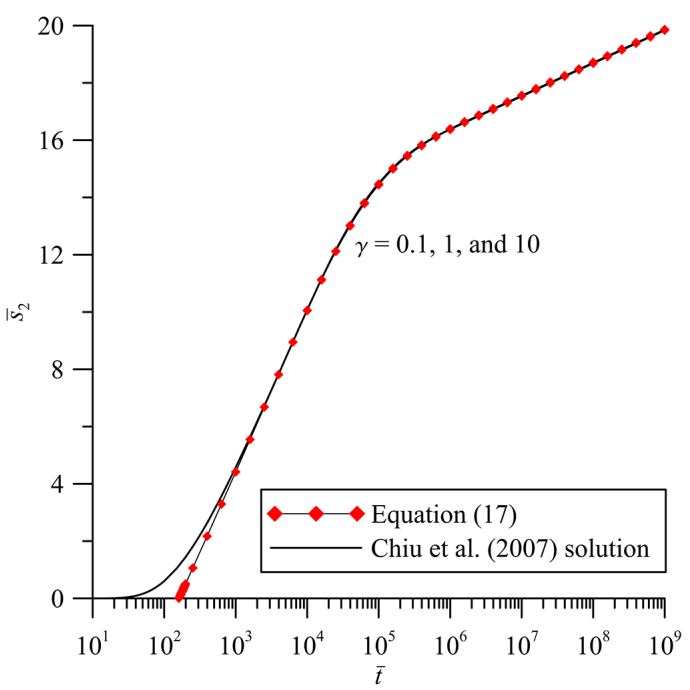

Figure 2. Predicted drawdowns by the Chiu et al. (2007) solution and the approximate solution, Eqs. (16) and (17), with $\gamma=0.1,1$, and 10 for (a) spatial distributions at $\bar{t}=3 \times 10^{6}$ and (b) temporal distributions at $\bar{r}=20$ with $\bar{z}=0.5, \bar{r}_{\mathrm{s}}=5, \bar{z}_{1}=0.4, \bar{z}_{2}=0.6$, and $\alpha_{1}=\alpha_{2}=10^{-7}$.

Note that Eqs. (19) and (20) are independent of $\bar{z}$, indicating that groundwater flow is only horizontal.

\subsection{Special case 2: solution for CFP at fully penetrating well in a homogeneous aquifer}

When $\bar{z}_{1}=0, \bar{z}_{2}=1$, and $\gamma=1$ (i.e., $z_{1}=0, z_{2}=b$, and $K_{\mathrm{r}_{1}}=K_{\mathrm{r}_{2}}$ ) for the case of a fully penetrating well in a homogeneous aquifer, Eqs. (16) and (17) yield

$s(\bar{r}, \bar{t})=\ln (\bar{R}(\bar{t}) / \bar{r})$ for $1 \leq \bar{r} \leq \bar{R}(\bar{t})$,

which is indeed a dimensionless form of Thiem's equation. Note that Eq. (21) can also be derived by substituting $\gamma=1$ into Eq. (19).

\section{Results and discussion}

\subsection{Accuracy of approximate solution}

On the basis of the comparison of predictions from the approximate solution and Chiu et al. (2007) Laplace-domain solution, we have concluded that the accuracy of the present solution depends only on dimensionless time $\bar{t}$ and radial distance $\bar{r}$ and does not relate to other dimensionless parameters and space variable. Consider representative parameters and variables as follows: $\bar{z}=0.5, \bar{r}_{\mathrm{s}}=5, \bar{z}_{1}=0.4, \bar{z}_{2}=0.6, \alpha_{1}=$ $\alpha_{2}=10^{-7}$, and $\gamma=0.1$ for positive skins, 1 for no skin and 10 for negative skins. Figure $2 \mathrm{a}$ shows the spatial drawdown distributions predicted by both solutions when $\bar{t}=3 \times 10^{6}$. The figure indicates that both solutions agree very well on the drawdown within the time-dependent radius of influence represented by $\bar{R}(\bar{t})$. The drawdown curves of $\gamma=0.1,1$ and 10 in the formation zone merge together at and beyond the interface, i.e., $\bar{r}_{\mathrm{s}}=5$, because of $\alpha_{1}=\alpha_{2}$. Figure $2 \mathrm{~b}$ displays the temporal drawdown distributions predicted by both solutions for an observation well at $\bar{r}=20$. This figure demonstrates that the drawdown curves also have a good match over the intermediate and late pumping periods. The discrepancy in dimensionless drawdown at the early period of $0 \leq \bar{t} \leq 600$ can be attributed to the absence of the time derivative term in both Eqs. (1) and (2). The drawdown dramatically increases at $\bar{t}=160$ as soon as $\bar{R}(\bar{t}=160)=20$. It seems reasonable to conclude that the approximate transient solution gives good predicted drawdown in an observation well over the entire pumping period except at an early time when the dynamic radius of influence reaches the well (i.e., $\bar{t} \cong 1.4(\bar{r}-1)^{2} / \pi$ derived by substituting $\bar{R}(\bar{t})=\bar{r}$ into Eq. (18) and rearranging the result).

\subsection{Vertical flow}

The vertical flow induced by well partial penetration is strongly dependent on both dimensionless lumped parameters $\alpha_{1} \bar{r}^{2}$ and $\alpha_{2} \bar{r}^{2}$ (i.e., $K_{z_{1}} r^{2} /\left(K_{\mathrm{r}_{1}} b^{2}\right)$ and $K_{z_{2}} r^{2} /\left(K_{\mathrm{r}_{2}} b^{2}\right)$, respectively). Figure 3 shows temporal drawdown distributions predicted by the approximate solution, Eq. (17), for $\alpha_{1}=\alpha_{2}$ ranging from $10^{-6}$ to $10^{-2}$ when $\bar{r}=10, \bar{z}=0.5, \bar{z}_{1}=0.4, \bar{z}_{2}=0.6, \bar{r}_{\mathrm{s}}=5$ and $\gamma=0.1$. Equation (20) is the drawdown solution for the CFP at a fully penetrating well; therefore, the vertical flow is absent. When $\alpha_{1} \bar{r}^{2}=\alpha_{2} \bar{r}^{2}=1$, the drawdown distributions predicted by both equations agree well, indicating that the vertical flow is negligible. We may, therefore, reasonably conclude that the vertical flow effect on the aquifer drawdown at an obser- 


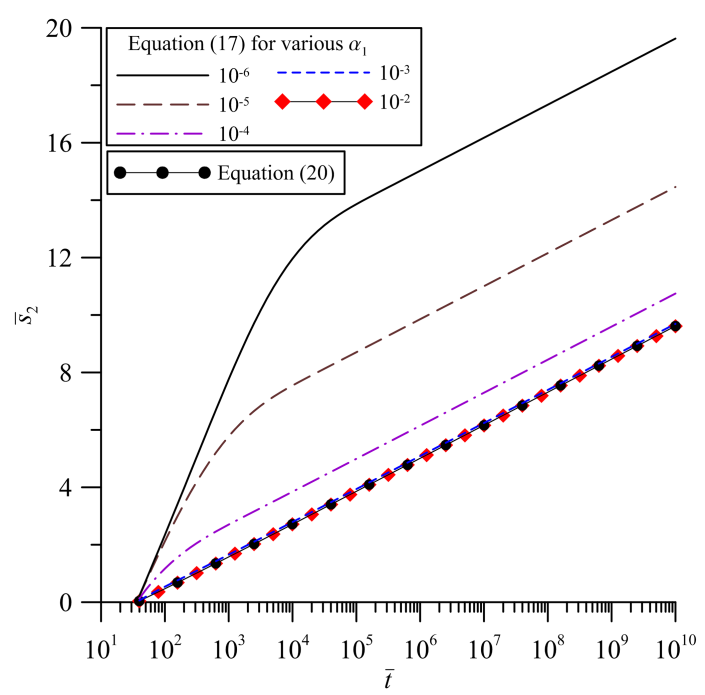

Figure 3. Temporal drawdown distributions predicted by the approximate solution, Eq. (17), with $\bar{r}=10, \bar{z}=0.5, \bar{z}_{1}=0.4$, $\bar{z}_{2}=0.6, \bar{r}_{\mathrm{S}}=5, \gamma=0.1$ and various values of $\alpha_{1}$ with $\alpha_{1}=\alpha_{2}$.

vation well vanishes when $\alpha_{1} \bar{r}^{2} \geq 1$ and $\alpha_{2} \bar{r}^{2} \geq 1$, i.e., $b$ is small, $r$ is large, and/or the values of $K_{z_{1}} / K_{\mathrm{r}_{1}}$ and $K_{z_{2}} / K_{\mathrm{r}_{2}}$ are large. On the other hand, Eq. (20) underestimates the drawdown induced by the CFP at a partially penetrating well because the vertical flow prevails when $\alpha_{1} \bar{r}^{2}<1$ or $\alpha_{2} \bar{r}^{2}<1$.

\section{Concluding remarks}

This study presents an approximate drawdown solution, Eqs. (16) and (17), in terms of a simple series for the CFP at a partially penetrating well in a radial two-zone confined aquifer. The solution is developed on the basis of the steadystate drawdown solution with an outer boundary represented by the time-dependent radius of influence. The comparison with the Chiu et al. (2007) solution reveals that the approximate solution gives accurate temporal drawdown distributions in an observation well over the entire pumping period except at an early time when the dynamic radius of influence reaches the well (i.e., $\bar{t} \cong 1.4(\bar{r}-1)^{2} / \pi$ derived by substituting $\bar{R}(\bar{t})=\bar{r}$ into Eq. (18) and rearranging the result). The analysis of the temporal drawdowns predicted by Eqs. (17) and (20) indicates that the vertical flow due to a partially penetrating well prevails under the conditions of thick aquifers, vicinity to the well, and/or small conductivity ratios (i.e., $\alpha_{1} \bar{r}^{2}<1$ or $\alpha_{2} \bar{r}^{2}<1$ ). Accordingly, conventional models neglecting the vertical flow will underestimate drawdown under those conditions. 


\section{Appendix A: Derivation of Eqs. (6) and (7)}

The finite Fourier cosine transform is defined, in our notation, as

$\hat{s}_{i}=\int_{0}^{1} \bar{s}_{i} \cos (n \pi \bar{z}) \mathrm{d} \bar{z}$,

where $i \in(1,2)$. The formula for the inverse transform is expressed as

$\bar{s}_{i}=\hat{s}_{i}(0)+2 \sum_{n=1}^{\infty} \hat{s}_{i}(n) \cos (n \pi \bar{z})$,

where $\hat{s}_{i}(n)$, a function of $n$, is the solution in the transform domain. Replacing $\bar{s}_{i}$ in Eq. (A1) by $\partial^{2} \bar{s}_{i} / \partial \bar{z}^{2}$ and applying integration by parts twice yields

$\int_{0}^{1} \frac{\partial^{2} \bar{s}_{i}}{\partial \bar{z}^{2}} \cos (n \pi \bar{z}) \mathrm{d} \bar{z}=\left.(-1)^{n} \frac{\partial \bar{s}_{i}}{\partial \bar{z}}\right|_{\bar{z}=1}-\left.\frac{\partial \bar{s}_{i}}{\partial \bar{z}}\right|_{\bar{z}=0}-(n \pi)^{2} \hat{s}_{i}$.

Applying the transform to Eqs. (1)-(5) on the basis of Eq. (A3) with $\partial \bar{s}_{i} / \partial \bar{z}=0$ results in the following equations:

$\frac{\partial^{2} \hat{s}_{1}}{\partial \bar{r}^{2}}+\frac{1}{\bar{r}} \frac{\partial \hat{s}_{1}}{\partial \bar{r}}-\lambda_{1}^{2} \hat{s}_{1}=0$ for $1 \leq \bar{r} \leq \bar{r}_{\mathrm{s}}$,

$\frac{\partial^{2} \hat{s}_{2}}{\partial \bar{r}^{2}}+\frac{1}{\bar{r}} \frac{\partial \hat{s}_{2}}{\partial \bar{r}}-\lambda_{2}^{2} \hat{s}_{2}=0$ for $\bar{r}_{\mathrm{s}} \leq \bar{r} \leq \bar{R}$,

$\hat{s}_{2}=0$ at $\bar{r}=\bar{R}$,

$\frac{\partial \hat{s}_{1}}{\partial \bar{r}}=-\gamma \omega / \varphi$ at $\bar{r}=1$,

$\hat{s}_{1}=\hat{s}_{2}$ at $\bar{r}=\bar{r}_{\mathrm{s}}$,

and

$\frac{\partial \hat{s}_{1}}{\partial \bar{r}}=\gamma \frac{\partial \hat{s}_{2}}{\partial \bar{r}}$ at $\bar{r}=\bar{r}_{\mathrm{s}}$.
The Fourier-domain solution of Eqs. (A4) and (A5) can be expressed as

$\hat{s}_{1}=c_{1} I_{0}\left(\lambda_{1} \bar{r}\right)+c_{2} K_{0}\left(\lambda_{1} \bar{r}\right)$,

and

$\hat{s}_{2}=c_{3} I_{0}\left(\lambda_{2} \bar{r}\right)+c_{4} K_{0}\left(\lambda_{2} \bar{r}\right)$,

where $I_{0}(\cdot)$ and $K_{0}(\cdot)$ are the modified Bessel functions of the first and second kinds of order zero, respectively, and $c_{1}, c_{2}, c_{3}$ and $c_{4}$ are undetermined coefficients. Substituting Eqs. (A10) and (A11) into Eqs. (A6)-(A9) and solving the four resultant equations leads to

$\left(c_{1}, c_{2}, c_{3}, c_{4}\right)=\left(\frac{\gamma \zeta \omega}{\varphi \lambda_{1} \psi}, \frac{\gamma \xi \omega}{\varphi \lambda_{1} \psi}, \frac{\gamma \omega K_{0}\left(\lambda_{2} \bar{R}\right)}{\varphi r_{\mathrm{s}} \lambda_{1} \psi},-\frac{\gamma \omega I_{0}\left(\lambda_{2} \bar{R}\right)}{\varphi r_{\mathrm{s}} \lambda_{1} \psi}\right)$,

(A12)

where $\psi, \zeta$ and $\xi$ are defined in Eqs. (10)-(12), respectively. According to Eq. (A12), Eqs. (A10) and (A11) can be written, respectively, as

$\hat{s}_{1}(\bar{r}, n)=\gamma F_{1}(\bar{r}, n) / \varphi$,

and

$\hat{s}_{2}(\bar{r}, n)=\gamma F_{2}(\bar{r}, n) /\left(\varphi \bar{r}_{\mathrm{s}}\right)$,

where $F_{1}(\bar{r}, n)$ and $F_{2}(\bar{r}, n)$ are defined in Eqs. (8) and (9), respectively. In the light of Eq. (A2), the inverse transforms to Eqs. (A13) and (A14) lead to Eqs. (6) and (7), respectively. Note that the first terms on the right-hand side of Eqs. (6) and (7) are derived via L'Hospital's law. 
Table A1. Summary of symbols used in the text and their definitions.

\begin{tabular}{ll}
\hline Symbols & Definitions \\
\hline$\left(s_{1}, s_{2}\right)$ & Drawdowns in skin and formation zones, respectively \\
$r$ & Radial distance from the center of the well \\
$r_{\mathrm{s}}$ & Radius of skin zone \\
$R$ & Radius of cylinder aquifer domain or the radius of influence \\
$\left(r_{\mathrm{W}}, r_{\mathrm{c}}\right)$ & Outer and inner radiuses of well, respectively \\
$z$ & Elevation from the aquifer bottom \\
$\left(z_{1}, z_{2}\right)$ & Lower and upper elevations of well screen, respectively \\
$t$ & Time since pumping \\
$b$ & Aquifer thickness \\
$Q$ & Pumping rate of well \\
$\left(K_{\mathrm{r}_{1}}, K_{\mathrm{r}_{2}}\right)$ & Radial hydraulic conductivities of skin and formation zones, respectively \\
$\left(K_{\mathrm{V}_{1}}, K_{\mathrm{V}_{2}}\right)$ & Vertical hydraulic conductivities of skin and formation zones, respectively \\
$S_{\mathrm{s}_{2}}$ & Specific storage of formation zone \\
$\left(T_{1}, T_{2}\right)$ & Transmissivities of skin and formation zones, respectively \\
$\left(\bar{s}_{1}, \bar{s}_{2}\right)$ & $\left(2 \pi T_{2} s_{1} / Q, 2 \pi T_{2} s_{2} / Q\right)$ \\
$\bar{t}$ & $K_{\mathrm{r}_{2}} t /\left(S_{\mathrm{S}_{2}} r_{\mathrm{w}^{2}}\right)$ \\
$\left(\bar{r}, \bar{r}_{\mathrm{s}}, \bar{R}\right)$ & $\left(r / r_{\mathrm{W}}, r_{\mathrm{S}} / r_{\mathrm{W}}, R / r_{\mathrm{W}}\right)$ \\
$\left(\bar{z}, \bar{z}_{1}, \bar{z}_{2}\right)$ & $\left(z / b, z_{1} / b, z_{2} / b\right)$ \\
$(\varphi, \gamma)$ & $\left(\bar{z}_{2}-\bar{z}_{1}, K_{\mathrm{r}_{2}} / K_{\mathrm{r}_{1}}\right)$ \\
$\left(\alpha_{1}, \alpha_{2}\right)$ & $\left(K_{z_{1}} r_{\mathrm{W}}^{2} /\left(K_{\mathrm{r}_{1}} b^{2}\right), K_{z_{2}} r_{\mathrm{W}}^{2} /\left(K_{\mathrm{r}_{2}} b^{2}\right)\right.$ \\
\hline
\end{tabular}


Acknowledgements. Research leading to this paper has been partially supported by the grants from the Taiwan Ministry of Science and Technology under the contract numbers NSC 1022221-E-009-072-MY2 and MOST 103-2221-E-009-156.

Edited by: S. Attinger

\section{References}

Carslaw, H. S. and Jaeger, J. C.: Conduction of heat in solids, 2nd Edn., Oxford University Press, London, 1959.

Chen, C. S.: A reinvestigation of the analytical solution for drawdown distributions in a finite confined aquifer, Water Resour. Res., 20, 1466-1468, 1984.

Chen, C. S. and Chang, C. C.: Theoretical evaluation of non-uniform skin effect on aquifer response under constant rate pumping, J. Hydrol., 317, 190-201, doi:10.1016/j.jhydrol.2005.05.017, 2006

Chiu, P. Y., Yeh, H. D., and Yang, S. Y.: A new solution for a partially penetrating constant-rate pumping well with a finitethickness skin, Int. J. Numer. Anal. Meth. Geomech., 31, 16591674, doi:10.1002/nag.607, 2007.

Fang, Y. M., Sun, J. J., and Chen, G. N.: A simple approach to the solution of the diffusion equation at the microcylinder electrode - an inspiration from the film projector, Chem. Phys. Chem., 10, 2393-2396, doi:10.1002/cphc.200900404, 2009.

Hantush, M. S.: Aquifer tests on partially penetrating wells, Trans. Am. Soc. Civil Eng., 127, 284-308, 1962.

Hemker, C. J.: Transient well flow in layered aquifer systems: the uniform well-face drawdown solution, J. Hydrol., 225, 19-44, 1999.

Kabala, Z. J. and El-Sayegh, H. K.: Transient flowmeter test: semianalytic crossflow model, Adv. Water Resour., 25, 103-121, 2002.

Novakowski, K. S.: A composite analytical model for analysis of pumping tests affected by wellbore storage and finite thickness skin, Water Resour. Res., 25, 1937-1946, 1989.
Papadopulos, I. S. and Cooper Jr., H. H.: Drawdown in a well of large diameter, Water Resour. Res., 3, 241-244, 1967.

Perina, T. and Lee, T. C.: General well function for pumping from a confined, leaky, unconfined aquifer, J. Hydrol., 317, 239-260, doi:10.1016/j.jhydrol.2005.05.020, 2006.

Theis, C. V.: The relation between the lowering of the piezometric surface and the rate and duration of discharge of a well using ground-water storage, Eos Trans. Am. Geophys. Union, 16, 519524, 1935.

Wang, C. T. and Yeh, H. D.: Obtaining the steady-state drawdown solutions of constant-head and constant-flux tests, Hydrol. Process., 22, 3456-3461, doi:10.1002/hyp.6950, 2008.

Wang, C. T., Yeh, H. D., and Tsai, C. S.: Transient drawdown solution for a constant pumping test in finite twozone confined aquifers, Hydrol. Earth Syst. Sci., 16, 441-449, doi:10.5194/hess-16-441-2012, 2012.

Wang, X., Xu, J., and Cai, Z.: Finite layer method for flow in layered radial two-zone aquifer systems, Ground Water, 50, 348354, doi:10.1111/j.1745-6584.2011.00849.x, 2012.

Yang, S. Y., Yeh, H. D., and Chiu, P. Y.: A closed form solution for constant flux pumping in a well under partial penetration condition, Water Resour. Res., 42, W05502, doi:10.1029/2004WR003889, 2006.

Yang, S. Y., Huang, C. S., Liu, C. H., and Yeh, H. D.: Approximate solution for a transient hydraulic head distribution induced by a constant-head test at a partially penetrating well in a twozone confined aquifer, J. Hydraul. Eng.-ASCE, 140, 04014030, doi:10.1061/(ASCE)HY.1943-7900.0000884, 2014.

Yeh, H. D. and Chang, Y. C.: Recent advances in modeling of well hydraulics, Adv. Water Resour., 51, 27-51, doi:10.1016/j.advwatres.2012.03.006, 2013.

Yeh, H. D., Yang, S. Y., and Peng, H. Y.: A new closed-form solution for a radial two-layer drawdown equation for groundwater under constant-flux pumping in a finite-radius well, Adv. Water Resour., 26, 747-757, doi:10.1016/S0309-1708(03)00046-0, 2003. 